\title{
Estudio de la progresión del cáncer de próstata incidental según el tipo de tratamiento aplicado
}

\author{
Napal Lecumberri $\mathrm{S}^{1}$, Lameiro Couso $\mathrm{FJ}^{2}$, Rubio Navarro $\mathrm{C}^{3}$, Gómez Dorronsoro $\mathrm{M}^{4}$, \\ Larrínaga Liñero $\mathrm{B}^{4}$, Ipiéns Aznar $\mathrm{A}^{1}$. \\ ${ }^{1}$ Servicio de Urología, ${ }^{2}$ Servicio de Medicina Preventiva y Gestión de la Calidad, \\ ${ }^{4}$ Servicio de Anatomía Patológica. Hospital de Navarra, Pamplona. ${ }^{3}$ Médico Becario Servicio Navarro de Salud.
}

Actas Urol Esp. 2007;31(8):810-818

\section{RESUMEN}

\section{ESTUDIO DE LA PROGRESIÓN DEL CÁNCER DE PRÓSTATA INCIDENTAL SEGÚN EL TIPO DE TRATAMIENTO APLICADO}

Introducción y objetivos: El cáncer de próstata es una neoplasia frecuente y con una carga de mortalidad significativa. Dado que se diagnostica a edades avanzadas y que con frecuencia crece lentamente, el tratamiento agresivo del cáncer de próstata localizado podría, en algunos casos, no aportar grandes beneficios. El estudio de la evolución de los cánceres de próstata incidentales diagnosticados hace 20 años podrían servir de utilidad para guiar las decisiones actuales.

Métodos: Análisis de supervivencia de los pacientes diagnosticados de cáncer de próstata incidental entre 1980 y 2000, con un seguimiento hasta abril de 2005. Se analizó la supervivencia global y la "cáncer específica", según el tipo de tratamiento y diversos factores anatomopatológicos.

Resultados: Se incluyeron 80 casos. De ellos, 34 fueron tratados con hormonoterapia, 4 con cirugia, 3 con radioterapia y 39 siguieron abstención vigilada. La supervivencia media fue de 8,87 años. En el grupo de bajo riesgo (Gleason menor de 6) la supervivencia cáncer-específica fue de 16 años y en el de alto riesgo (Gleason mayor de 7) de 6 años, sin diferencias por tratamiento.

Conclusiones: En el grupo de bajo riesgo la abstención vigilada resulta una alternativa aceptable. Esta alternativa puede no ser válida en los de alto riesgo. La hormonoterapia no parece aportar beneficio alguno en los cánceres de próstata incidentales.

Palabras clave: Hallazgo incidental. Cáncer de próstata. Análisis de supervivencia.

\begin{abstract}
A STUDY OF THE PROGRESSION OF THE INCIDENTAL PROSTATE CANCER DEPENDING ON THE KIND OF TREATMENT PERFORMED

Introduction and objectives: Prostate cancer is a common neoplasm, with a significant burden of mortality. Since it's diagnosed at old ages and usually growths slowly, agresive treatment of localised cancer of prostate could be of little benefit. The study of the evolution of incidental prostate cancer diagnosed 20 years ago could give directions for present decision-making

Methods: We performed a time survival analysis of patients diagnosed of incidental prostate cancer between 1980 and 2000, and followed them until april 2005. We analysed overall and disease-specific mortality, related to the kind of treatment and some anatomopathological characteristics.

Results: We included 80 cases. Treatment was hormonotherapy in 34 cases, surgery in 4 , radiotherapy in 3 and watchful waiting in 39. Overall average survival was 8.87 years. Disease-specific survival in low risk group (Gleason lower than 6) was 16 years, and in high risk group (Gleason higher than 7) was 6 years. No differences were found between hormonotherapy and watchful waiting.

Conclusions: While in low risk patients watchful waiting represents an acceptable aproaching, it could not be adequate in high risk cases. Hormonotherapy showed no benefit in incidental prostate cancer.
\end{abstract}

Keywords: Incidental findings. Prostatic neoplasms. Survival analysis.

Proyecto de Investigación número 41/2004 del Departamento de Salud del Gobierno de Navarra. 
$\mathrm{E}^{1}$ cáncer de próstata (CP) es una de las neoplasias masculinas más frecuentes en Occidente, tanto en incidencia como en mortali$\operatorname{dad}^{1-5}$.

Su aparición está muy relacionada con la edad, de modo que su incidencia es máxima en la octava década de la vida ${ }^{6,7}$. Por otra parte, los estudios de necropsias demuestran la existencia de focos tumorales prostáticos en gran cantidad de los varones muertos por otra causa y esto incluyendo a todos los grupos de edad ${ }^{8}$. Puede afirmarse que el CP afectará histológicamente a casi la mitad de los varones, aunque luego tenga en muchos casos, un crecimiento muy lento y un período preclínico excepcionalmente largo.

Desde finales de los años ochenta del pasado siglo, la generalización del Antígeno Prostático Específico (acrónimo en inglés PSA) como marcador tumoral de esta neoplasia ha supuesto un aumento significativo de los casos diagnosticados en estadios localizados. Esto supone que los casos se tratan, en general, en estadios más precoces de la enfermedad. Actualmente, en el momento del diagnóstico, la enfermedad presenta afectación extraprostática entre el 30\% y el $50 \%$ de los pacientes, con una supervivencia media en estos casos de alrededor de 24 meses $^{9}$. Por el contrario, si la enfermedad es "órgano confinada” en el momento del diagnóstico, la progresión puede demorarse muchos años ${ }^{10}$.

La disparidad entre la incidencia histológica y la clínica del $\mathrm{CP}$, sumado al hecho de la pobre especificidad del PSA, ha permitido que se plantee en círculos cada vez más extensos del mundo médico la hipótesis de que algunas neoplasias de próstata diagnosticadas precozmente mediante búsqueda activa están siendo "sobre tratadas", dado que su lenta evolución pudiera hacer que su tratamiento no supusiera beneficio alguno frente a una actitud expectante. Es ésta una cuestión crucial, ya que una respuesta afirmativa exigiría de inmediato un replanteamiento de la actitud terapéutica frente a los CP, asî diagnosticados, al igual que sobre la idoneidad de generalizar los referidos medios diagnósticos basados fundamentalmente en la determinación sistemática del PSA en varones sin sintomatología prostática ${ }^{11-13}$.
Para determinar el beneficio que ofrece el diagnóstico y tratamiento precoz del CP así diagnosticado, se han puesto en marcha varios estudios prospectivos de gran envergadura ${ }^{14,17}$. Mientras llegan los resultados de los mismos, persisten las dudas referidas tanto a la conveniencia y beneficios obtenidos mediante la determinación indiscriminada del PSA en varones sin ninguna sintomatología, para diagnosticar de forma precoz el $\mathrm{CP}$, así como la mejor actitud terapéutica en los casos "órgano confinados" detectados mediante este proceder.

Antes de la aparición y uso prácticamente masivo del PSA, era frecuente el hallazgo anatomopatológico de $\mathrm{CP}$ en muestras histológicas obtenidas tras el tratamiento de una supuesta hiperplasia benigna de próstata (HBP) sin sospecha de neoplasia. El seguimiento y estudio de estos casos - conocidos como CP incidentales - permite en el momento presente, con las grandes limitaciones de todo estudio retrospectivo, la posibilidad de estudiar con una perspectiva de 20 años, la evolución seguida por varones a los cuales se les diagnosticó un CP en estas circunstancias, así como valorar algunos de los factores que pudieron influir en su evolución.

De este modo y con los datos expuestos, el objetivo del presente estudio es determinar la supervivencia, así como los factores que pudieran influir sobre ella y que, en cierto modo, actuarían como elementos pronósticos de aquellos pacientes diagnosticados de CP incidental, al tiempo que se compara la evolución de estos casos, una vez realizado el diagnóstico en función de la pauta de actuación seguida tras conocer la existencia de focos insospechados de "adenocarcinoma” en las piezas quirúrgicas.

\section{PACIENTES Y MÉTODOS}

Se definió como "cáncer incidental de próstata” el CP diagnosticado anatomopatológicamente sobre piezas obtenidas mediante adenomectomía de próstata o resección transuretral (RTU) de la misma, en pacientes diagnosticados de HBP, en los cuales no existía sospecha de carcinoma prostático. Con este criterio, estos tumores se corresponden con los estadios Tla y $\mathrm{T} 1 \mathrm{~b}$ de la clasificación TNM de la UICC de 1997.

Los casos de cáncer incidental de próstata se obtuvieron de la base de datos informatizada del 
Servicio de Anatomía Patológica del Hospital de Navarra. Esta base de datos recoge los resultados de las muestras examinadas entre los años 1980 y 2000, en formato de texto libre, es decir, no codificado. Para recuperar todos los posibles CP incidentales, se realizaron diversas consultas combinando el término "adenocarcinoma" con diversas palabras sugestivas de HBP (Tabla 1).

Tabla 1. Estrategia de búsqueda

ADENOCAR\$ AND PROST\$ AND RETENCIO\$
ADENOCAR\$ AND PROST\$ AND SOND\$
ADENOCAR\$ AND PROST\$ AND OBSTRUC\$
ADENOCA\$ AND PROST\$ AND RTU
ADENOCA\$ AND PROST\$ AND GLOBO
ADENOCA\$ AND PROST\$ AND PROSTATISMO
ADENOCA\$ AND PROST\$ AND HBP
ADENOCA\$ AND PROST\$ AND HIPERPLAS\$
Notas
- El carácter \$ indica el comodín para las búsquedas en la base
de datos informática
- La palabra AND indica el operador booleano.

Las historias de los pacientes se revisaron para obtener la información clínica relevante, y se consignaron los datos correspondientes en un formulario creado específicamente para tal fin. Se consideraron las siguientes variables clínicas: edad en el momento del diagnóstico, tratamiento de la HBP (adenomectomía o RTU prostática), fecha del diagnóstico de $\mathrm{CP}$, tratamiento adicional tras el diagnóstico de $\mathrm{CP}$ y revisiones en consulta urológica y sus fechas.

Además, las piezas anatomopatológicas correspondientes fueron revisadas de nuevo por dos médicos especialistas del Servicio de Anatomía Patológica para determinar las siguientes variables de interés: Peso del tejido extirpado, porcentaje de cristales con tumor, porcentaje de tejido afectado por el tumor, tipo histológico, índice Gleason, presencia de invasión perineural, positividad al marcador P53 e índice de proliferación del marcador ki67.

Por otra parte se recuperaron del registro informatizado del Laboratorio de Bioquímica las determinaciones de PSA que pudieran haberse realizado a los pacientes incluidos en el estudio.

Así pues, entraron en el seguimiento los pacientes que cumplieron los criterios de "inci- dentalomas" desde 1980 hasta 2000. El cierre del seguimiento se estableció el 30 de abril de 2005.

El estado vital de los pacientes al cierre del estudio, y, en su caso, la fecha y la causa de la defunción se consultaron a los registros de mortalidad y cáncer del Instituto de Salud Pública de Navarra. En estos registros la causa de la muerte figura codificada según la clasificación CIE, en sus versiones 9 (hasta mediados de 1999) ó 10 (desde entonces). La muerte se atribuyó al CP en los pacientes en cuya causa de defunción constaban los códigos correspondientes (185 para la CIE 9 y C61 para la CIE 10).

Los datos se analizaron con el programa estadístico SPSS $®$. Para comparar las variables independientes categóricas se empleó la prueba de "ji cuadrado". Para comparar las variables independientes numéricas se usó la prueba "t de Student" para muestras independientes. Se definieron dos tipos de supervivencia: la global (tiempo transcurrido entre el diagnóstico de CP y el fallecimiento por cualquier causa) y la causa específica (tiempo entre el diagnóstico y la defunción por cáncer de próstata). Ambas se determinaron mediante el "método de Kaplan-Meier" y se empleó la prueba de "log-rank" para comparar curvas según los tipos de tratamiento.

\section{RESULTADOS}

De los 141 posibles casos de cáncer de próstata incidental, 40 se excluyeron por constatación de posible sospecha de malignidad (aunque esta fuera remota) previa a la intervención en la revisión de la historia clínica; trece por no cumplir criterios anatomopatológicos; siete por no disponer de historia clínica; un registro por no existir ni una sola revisión de seguimiento tras la intervención.

Resultaron válidos para el análisis, 80 registros.

La edad media en el momento del diagnóstico fue de 74,33 años. El procedimiento empleado para tratar la HBP fue la adenomectomía abierta en 43 casos y la RTU en los 37 restantes. En 39 casos no se administró ningún tratamiento adicional tras el diagnóstico de cáncer incidental, en 34 se pautó hormonoterapia, cuatro pacientes fueron sometidos cirugía radical de la próstata y otros tres a radioterapia. En el estudio anatomo- 
patológico, 33 pacientes (41\%) presentaron un índice de Gleason mayor de 6; 8 pacientes $(10 \%)$ mostraron presencia de invasión perineural y 5 pacientes (6\%) presentaron positividad al marcador P53. El peso del tejido extirpado en la intervención para tratar la HBP fue en promedio de 35 gramos, y la estimación media de la proporción de tejido afectado por el tumor fue de $25 \%$. En los 18 casos en los que se determinó el PSA antes de la intervención para tratar la HBP, el valor medio del PSA fue de 3,27. Durante todo el seguimiento, el promedio de revisiones urológicas fue de 3,15 (Tablas 2 y 3 ).

Tabla 2. Características de los 80 pacientes incluidos

\begin{tabular}{|c|c|c|}
\hline Intervención realizada para & $\mathbf{N}$ & $\%$ \\
\hline Adenomectomía & 43 & $54 \%$ \\
\hline Resección transuretral & 37 & $46 \%$ \\
\hline \multicolumn{3}{|l|}{$\begin{array}{l}\text { Tratamiento adicional aplicado } \\
\text { tras el diagnóstico de cáncer }\end{array}$} \\
\hline Hormonoterapia & 34 & $43 \%$ \\
\hline Cirugía & 4 & $5 \%$ \\
\hline Radioterapia & 3 & $4 \%$ \\
\hline Ninguno & 39 & $49 \%$ \\
\hline \multicolumn{3}{|l|}{ Índice de Gleason } \\
\hline 2 a 6 & 47 & $59 \%$ \\
\hline 7 A 10 & 33 & $41 \%$ \\
\hline \multicolumn{3}{|l|}{$\begin{array}{l}\text { Presencia de invasión perineural } \\
\text { en la biopsia }\end{array}$} \\
\hline NO & 72 & $90 \%$ \\
\hline SI & 8 & $10 \%$ \\
\hline \multicolumn{3}{|l|}{ Tipo histológico } \\
\hline Adenocarcinoma convencional & 78 & $98 \%$ \\
\hline Adenocarcinoma de los ductos & 2 & $3 \%$ \\
\hline \multicolumn{3}{|l|}{ Positividad P53 } \\
\hline NO & 71 & $89 \%$ \\
\hline SI & 5 & $6 \%$ \\
\hline No determinable por falta de material & 4 & $5 \%$ \\
\hline
\end{tabular}

La fecha del cierre del seguimiento fue el 30 de abril de 2005. En este punto, los casos podian presentar uno de los siguientes estados vitales: muerto por cáncer de próstata, muerto por otra causa, perdido o retirado vivo al final del seguimiento. En la Tabla 4 se muestran estos estados vitales en relación con la actitud terapéutica adoptada tras el hallazgo de focos de adenocarcinoma en el material extirpado.

La supervivencia media desde el diagnóstico hasta la defunción por cualquier causa fue de casi 9 años. La mediana de supervivencia, esto es, el tiempo transcurrido desde el diagnóstico hasta el momento en que la mitad de las 80 personas incluidas fallecieron fue de 11 años (Tabla 5 y Fig. 1).

Para estudiar el efecto de las distintas actitudes terapéuticas sobre la supervivencia, dado el escaso número de pacientes tratados con radioterapia complementaria (tres) y con cirugia radical (cuatro), se optó por comparar solo los casos tratados con hormonoterapia con aquellos en los cuales se asumió una actitud expectante. Ambos grupos no mostraron diferencias en cuanto a la intervención realizada para tratar la HBP, la presencia de invasión perineural en la biopsia, la proliferación del ki67, la edad en el momento del diagnóstico, el peso del tejido extirpado ni el número de revisiones realizadas por el urólogo durante el período de seguimiento. Sin embargo, si hubo diferencias en otras variables. Así, el grupo tratado con hormonoterapia mostró con mayor frecuencia valor del índice Gleason superior a 6 , positividad $\mathrm{p} 53$, porcentaje de tejido afectado mayor del $50 \%$ y porcentaje de cristales con afectación tumoral del 100\% (Tablas 6 y 7).

A lo largo del seguimiento, en el grupo no tratado se produjeron 18 fallecimientos, de ellos 5

Tabla 3. Características de los 80 pacientes incluidos (continuación)

\begin{tabular}{|c|c|c|c|c|c|}
\hline & $\begin{array}{c}\text { Pacientes para los } \\
\text { que hay datos }\end{array}$ & Minimo & Máximo & Media & $\begin{array}{c}\text { Desviación } \\
\text { estándar }\end{array}$ \\
\hline Edad en el momento del diagnóstico & 80 & 53,85 & 95,68 & 74,33 & 6,67 \\
\hline Valor de PSA basal & 18 & 1,01 & 4,00 & 3,27 & 0,59 \\
\hline Porcentaje de tejido afectado del total resecado & 80 & 1 & 96 & 25 & 28 \\
\hline Peso del tejido extirpado (gramos) & 60 & 3,00 & 100,00 & 35,18 & 21,77 \\
\hline Índice de proliferación del marcador k67 (porcentaje) & 76 & 0 & 34 & 8 & 8 \\
\hline Porcentaje de cristales vistos con afectación tumoral & 80 & 4 & 100 & 53 & 37 \\
\hline Número de revisiones realizadas durante el seguimiento & 80 & 0 & 12 & 3,16 & 2,89 \\
\hline
\end{tabular}


Tabla 4. Estado vital de los pacientes al cierre del seguimiento, por tipo de tratamiento

\begin{tabular}{|c|c|c|c|c|}
\hline & $\begin{array}{c}\text { No tratados } \\
n\end{array}$ & $\begin{array}{c}\text { Hormonoterapia } \\
\text { n }\end{array}$ & $\begin{array}{c}\text { Radioterapia } \\
\mathbf{n}\end{array}$ & $\begin{array}{c}\text { Cirugia } \\
\mathbf{n}\end{array}$ \\
\hline Muerto por cáncer de próstata & 5 & 11 & 1 & 0 \\
\hline Muerto por otras causas & 13 & 11 & 12 & 1 \\
\hline Perdido antes del cierre & 0 & 4 & 0 & 0 \\
\hline Vivo al cierre del seguimiento & 21 & 8 & 0 & 3 \\
\hline TOTAL & 39 & 34 & 3 & 4 \\
\hline
\end{tabular}

Tabla 5. Supervivencia de todos los pacientes hasta la muerte por cualquier causa

\begin{tabular}{lc}
\hline Número de casos & 80 \\
Tiempos incompletos* & 36 \\
Tiempos completos* & 44 \\
Media de supervivencia, años & 8,87 \\
Intervalo de confianza 95\% & 7,37 a 0,37 \\
Mediana de supervivencia, años & 10,99 \\
Intervalo de confianza 95\% & 6,22 a 15,16 \\
\hline *"Tiempos incompletos" son los generados por los pacientes per- \\
didos durante el seguimiento. \\
**Tiempos incompletos" son los generados por los pacientes \\
fallecidos por cualquier causa.
\end{tabular}

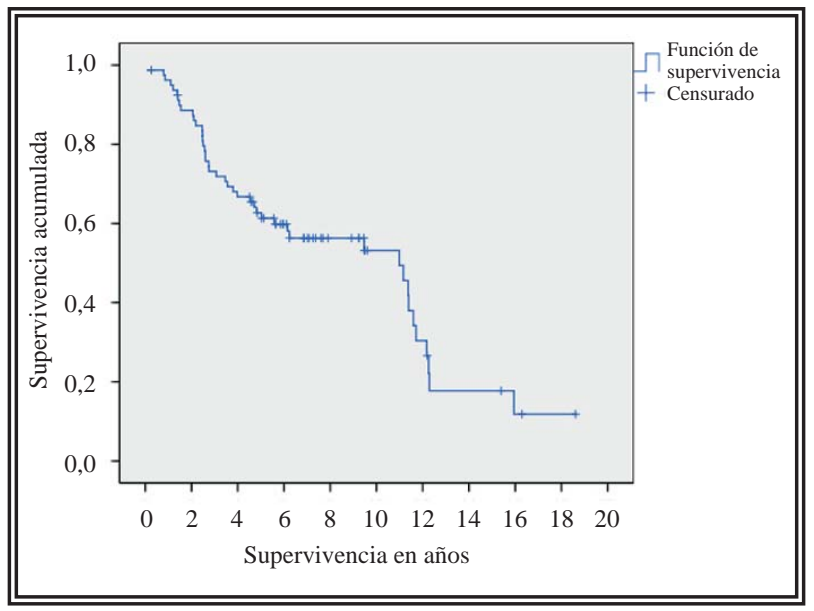

FIGURA 1. Función de supervivencia hasta la muerte por cualquier causa.

por CP y 13 por otras causas. En el grupo de tratados con hormonoterapia, los fallecimientos registrados fueron 22,11 por $\mathrm{CP}$ y otros 11 por otras causas. La supervivencia media hasta el fallecimiento por CP fue de casi 12 años en el grupo de la hormonoterapia y 14 en el de los no tratados, con significación estadística de esta diferencia (Tabla 8 y Fig. 2).

Se realizó un análisis de la supervivencia hasta la muerte por CP estratificado por el índice
Gleason para cada grupo de tratamiento. Se produjeron tres muertes por CP en el grupo de bajo riesgo (Gleason menor o igual a 6) y 13 en el de riesgo elevado (Gleason mayor o igual a 7). La supervivencia media fue de unos 16 años en el grupo de bajo riesgo y de unos 6 años en el de riesgo elevado (Tabla 9 y Figs. 3 y 4).

\section{DISCUSIÓN Y CONCLUSIONES}

El objetivo de este estudio era determinar el intervalo de tiempo hasta la muerte del CP incidental con y sin tratamiento complementario y según los distintos marcadores de riesgo identificados en el análisis anatomopatológico de las piezas de resección.

El carácter retrospectivo del presente estudio suponía la incógnita del número de casos que se iban a encontrar, así como el que las características de los mismos permitieran explorar todas las cuestiones planteadas. En este sentido, el número final de pacientes seguidos - ochenta no aporta potencia estadística suficiente para algunos análisis estratificados finos, que permitan determinar con claridad el papel de los marcadores de riesgo anatomopatológicos. Del mismo modo, las características de los casos recuperados no permiten ciertas comparaciones interesantes que se plantearon al diseñar el estudio. Por ejemplo, el hecho de encontrar tan solo 3 y 4 pacientes tratados respectivamente con radioterapia y cirugía radical impide la comparación de ambas alternativas terapéuticas con la abstención vigilada. No obstante, el número de casos incluido finalmente -ochenta- sí que permite obtener algunas conclusiones interesantes y aplicables al entorno clínico en el cual el estudio se plantea, esto es, la conveniencia de determinar el nivel de PSA en varones mayores de 50 años que acuden a la consulta del urólogo sin sospecha de $\mathrm{CP}$ y la actitud ante un CP estadio I. 
Tabla 6. Comparación de diversos factores de riesgo entre pacientes no tratados y pacientes tratados con hormonoterapia

\begin{tabular}{|c|c|c|c|c|}
\hline & $\begin{array}{c}\text { No } \\
\text { tratados }\end{array}$ & Hormonoterapia & Valor de $\mathbf{p}^{*}$ & $\begin{array}{l}\text { Odd ratio (intervalo } \\
\text { de confianza } 95 \% \text { ) }\end{array}$ \\
\hline \multicolumn{5}{|c|}{ Intervención realizada para el tratamiento de la $\mathrm{HBP}$} \\
\hline Adenomectomía & 26 & 16 & & \\
\hline Resección transuretral & 13 & 18 & 0,10 & $0,44(0,17-1,15)$ \\
\hline \multicolumn{5}{|l|}{ Índice Gleason } \\
\hline 2 a 6 & 31 & 13 & & \\
\hline 7 a 10 & 8 & 21 & 0,00 & $0,16(0,06-0,45)$ \\
\hline \multicolumn{5}{|c|}{ Presencia de invasión perineural en la biopsia } \\
\hline NO & 36 & 30 & & \\
\hline SI & 3 & 4 & 0,56 & $0,62(0,13-3,01)$ \\
\hline \multicolumn{5}{|l|}{ Positividad P53 } \\
\hline NO & 35 & 30 & & \\
\hline SI & 0 & 4 & 0,04 & $0,46(0,36-0,60)$ \\
\hline \multicolumn{5}{|l|}{ Porcentaje de tejido afectado } \\
\hline Menor del 50\% & 37 & 22 & & \\
\hline Mayor del 50\% & 2 & 12 & 0,00 & $0,09(0,02-0,49)$ \\
\hline \multicolumn{5}{|l|}{ Índice de proliferación del marcador ki67 } \\
\hline No proliferación $(<=3,5 \%)$ & 15 & 11 & & \\
\hline Proliferación mayor de $3,5 \%$ & 20 & 23 & 0,37 & $0,63(0,24-1,70)$ \\
\hline \multicolumn{5}{|l|}{ Porcentaje de cristales con tumor } \\
\hline Menor del $100 \%$ & 35 & 15 & & \\
\hline $100 \%$ & 4 & 19 & 0,00 & $0,90(0,02-0,31)$ \\
\hline
\end{tabular}

*Ji cuadrado.

Tabla 7. Comparación de diversos factores de riesgo entre pacientes no tratados y pacientes tratados con hormonoterapia (continuación)

\begin{tabular}{|c|c|c|c|c|}
\hline & $\begin{array}{c}\text { No } \\
\text { tratados }\end{array}$ & Hormonoterapia & Valor de $\mathbf{p}^{*}$ & $\begin{array}{c}\text { Diferencia de } \\
\text { medias (intervalo } \\
\text { de confianza 95\%) }\end{array}$ \\
\hline \multicolumn{5}{|l|}{ Edad en el momento del diagnóstico } \\
\hline $\begin{array}{l}\text { Número de pacientes para los que } \\
\text { hay datos }\end{array}$ & 39 & 34 & & \\
\hline Valor promedio & 73,84 & 76,20 & 0,12 & $-2,35(-5,30-0,60)$ \\
\hline \multicolumn{5}{|l|}{ Peso del tejido extirpado (gramos) } \\
\hline Número de pacientes para los que hay datos & 34 & 23 & & \\
\hline Valor promedio & 35,32 & 32,70 & 0,65 & $-2,63(-9,03-14,28)$ \\
\hline \multicolumn{5}{|l|}{$\begin{array}{l}\text { Número de revisiones que realizó el paciente } \\
\text { durante el seguimiento }\end{array}$} \\
\hline Número de pacientes para los que hay datos & 39 & 34 & & \\
\hline Valor promedio & 2,62 & 2,97 & 0,57 & $-0,35(-1,58-0,87)$ \\
\hline
\end{tabular}

*Estadístico T Student.

El primer elemento relevante al hablar de supervivencia, y más en el caso del $\mathrm{CP}$, es la edad. La media de edad en el momento del diagnóstico fue de 74,33 años. Holmberg, en Suecia, comunica edades sensiblemente inferiores ${ }^{18}$ (65 años), mientras que en España, otros autores han encontrado edades similares a las del presente estudio ${ }^{19}$ (75,2 años).

Otro elemento relevante es el valor de PSA antes de la intervención para tratar la HBP. La presente serie arranca en 1980, es decir, varios años antes de la generalización del uso del PSA en la práctica urológica. En consecuencia, solo 18 casos cuentan con determinaciones de PSA previas a la intervención. La media de PSA en el momento del diagnóstico fue de 3,27 , es decir, en el rango considerado en nuestro entorno como zona gris.

En cuanto al índice Gleason, casi el 60\% de los pacientes considerados presentó valores de bajo riesgo (Gleason menor o igual a 6), frente a un 40 que los presentó de riesgo elevado (Gleason mayor o igual a 7). De los 80 pacientes tan solo 
Tabla 8. Supervivencia hasta la muerte por CP de los pacientes tratados con hormonoterapia comparada con la de los no tratados.

\begin{tabular}{lcc}
\hline & $\begin{array}{c}\text { No } \\
\text { tratados }\end{array}$ & Hormonoterapia \\
\hline Número de casos & 39 & 34 \\
Tiempos incompletos* & 34 & 23 \\
Tiempos completos** & 5 & 11 \\
Media de supervivencia, años & 14,34 & 11,86 \\
Intervalo de confianza 95\% & 12,74 a 15,93 & 8,76 a 14,96 \\
Prueba de log-rank & 5,20 & \\
Significación & 0,02 & \\
\hline
\end{tabular}

*"Tiempos incompletos" con los generados por los pacientes perdidos durante el seguimiento, vivos el día 30 de abril de 2005 o fallecidos por causa distinta del $\mathrm{CP}$

**"Tiempos completos" son los generados por los pacientes fallecidos por $\mathrm{CP}$

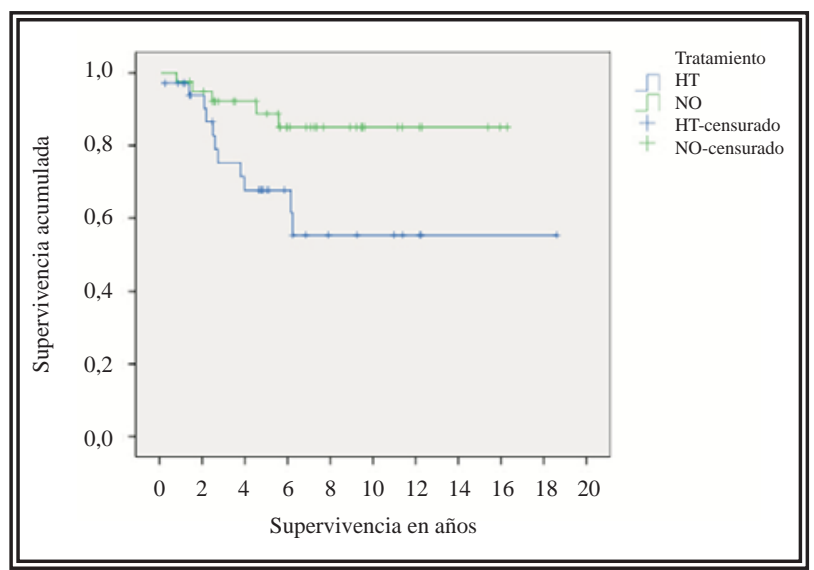

FIGURA 2. Función de supervivencia hasta la muerte por CP según actitud terapéutica.

resultaron perdidos durante el seguimiento 4 , es decir, un 5\%, lo cual supone un criterio de buena calidad de los datos. Cuarenta y cuatro personas fallecieron durante el estudio, con una supervivencia media desde el diagnóstico de 8,87 años.

La comparación de la supervivencia cáncerespecífica entre pacientes tratados con hormonoterapia y pacientes no tratados arroja resultados favorables al no tratamiento. En efecto, aparece un efecto negativo de la hormonoterapia, ya que este grupo registra más muertes (11 frente a $5 \mathrm{de}$ los no tratados) y 3 años menos de supervivencia.

La primera hipótesis que se consideró para explicar esta observación fue la existencia de un sesgo de información. El supuesto fue el siguiente: los pacientes no tratados fueron dados por curados y no siguieron revisiones por el urólogo. De este modo, en el momento de la muerte, el médico que firmó el diagnóstico de defunción no
Tabla 9. Supervivencia hasta la muerte por CP de los pacientes tratados con hormonoterapia comparada con la de los no tratados, estratificado por índice Gleason

\begin{tabular}{lcc}
\hline Índice Gleason & $\begin{array}{c}\text { No } \\
\text { tratados }\end{array}$ & Hormonoterapia \\
\hline Menor o igual a 6 & & \\
Número de casos & 31 & 13 \\
Tiempos incompletos & 29 & 12 \\
Tiempos completos & 2 & 1 \\
Media de supervivencia, años & 15,33 & 17,38 \\
Intervalo de confianza 95\% & 14,04 a 16,62 & 15,06 a 19,70 \\
Prueba de log-rank & 0,01 & \\
Significación & 0,92 & \\
\hline
\end{tabular}

\section{Mayor o igual a 7}

Número de casos

Tiempos incompletos

Tiempos completos

Media de supervivencia, años

Intervalo de confianza 95\%

Prueba de log-rank

21

$5 \quad 11$

$3 \quad 10$

$7,26 \quad 5,68$

Significación

0,41

*"Tiempos incompletos" son los generados por los pacientes perdidos durante el seguimiento, vivos el día 30 de abril de 2005 o fallecidos por causa distinta del CP

**"Tiempos completos" son los generados por los pacientes fallecidos por $\mathrm{CP}$

la achacó a la existencia de un CP evolucionado. Sin embrago, en el caso de los pacientes en tratamiento hormonoterápico, el seguimiento urológico fue mejor cumplimentado y, por lo tanto, el diagnóstico de $\mathrm{CP}$ se tuvo presente en el momento de extender el certificado de defunción. Para contrastar esta hipótesis se compararon las revisiones urológicas consignadas en las historias clínicas de los pacientes. El hecho de que en ambos grupos el número de revisiones sea similar (3, en números redondos) refuta la hipótesis.

Otra explicación es la distinta edad en ambos grupos. El grupo no tratado es unos dos años y medio más joven que el tratado. A pesar de que la diferencia no es estadísticamente significativa, no se puede obviar el hecho de que la significación estadística está relacionada con el número de casos estudiados, y que éste es, como se ha dicho más arriba, poco numeroso. Además, el estudio de la supervivencia "cáncer-específica" muestra un intervalo hasta la muerte de unos dos años y medio mayor en los no tratados que en los tratados, de modo que, como el diagnóstico en los no tratados es anterior tardan un poco más en morir, pero acaban falleciendo aproximadamente a la misma edad. 


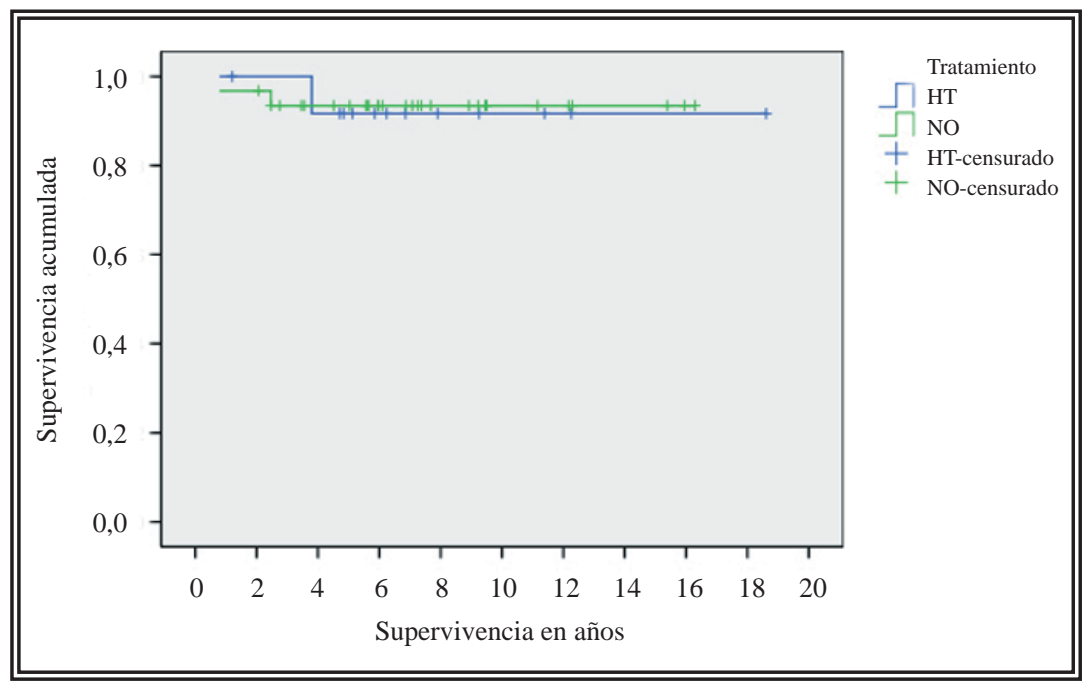

FIGURA 3. Función de supervivencia hasta la muerte por CP según actitud terapéutica. Gleason menos o igual a 6.

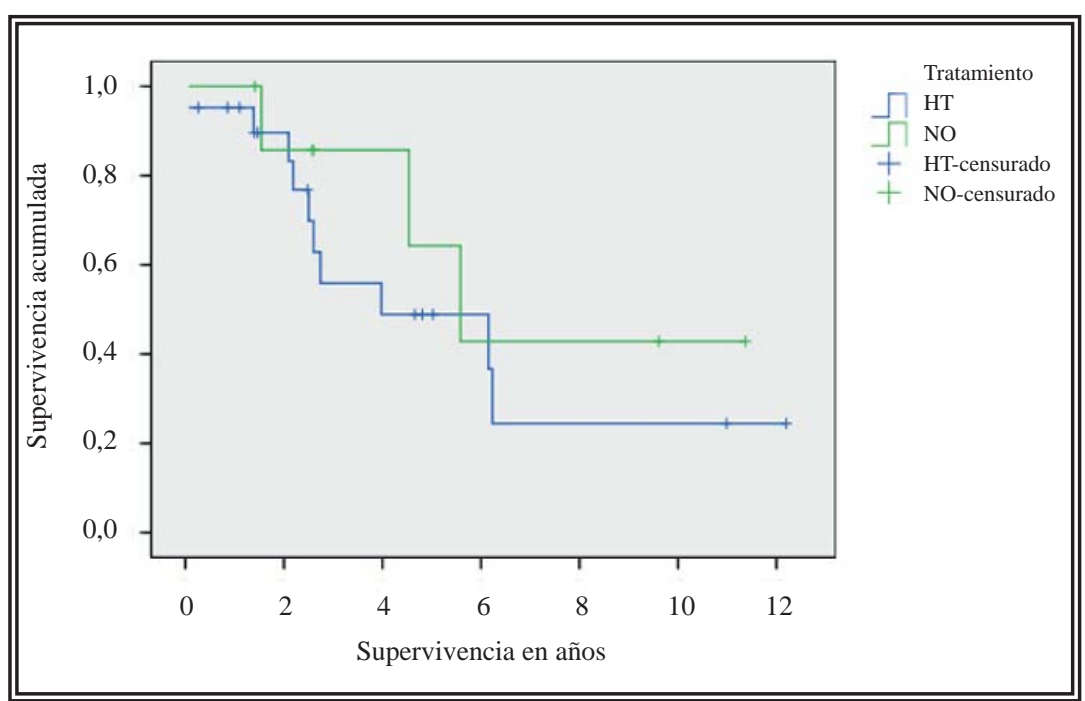

FIGURA 4. Función de supervivencia hasta la muerte por CP según actitud terapéutica. Gleason mayo o igual a 7.

Además de esta explicación, lo más relevante es la distribución de los factores de riesgo en ambos grupos. Si bien hay ciertos factores en los cuales ambos grupos no difieren - o al menos no difieren con significación estadística - sí que hay marcadores de riesgo importantes que se observan con más frecuencia en el grupo de tratamiento. Así, por cada paciente con Gleason mayor de 6 que se trata, tan solo lo hace 0,16 pacientes con Gleason menor de 7. Del mismo modo, los pacientes del grupo de la hormonoterapia presentan más tejido afectado, más cristales con tumor y más positividad al P53. Se puede concluir de ello que, sin que existiese en nuestro centro en aquel momento - recordemos que estamos hablando de casos clínicos de hace entre 25 y cinco años - un protocolo o pauta de actuación expresa frente al cáncer incidental de próstata, los urólogos adoptaban una actitud más agresiva - añadir la terapia hormonal - en los casos informados anatomopatológicamente como de más riesgo de proliferar: mayor índice Gleason, más tejido afectado, marcadores positivos. Es decir, los del grupo tratado tenían "a priori”, peor pronóstico.

En consecuencia, se realizó un análisis estratificado para diferenciar entre el efecto de los factores de mal pronóstico -en concreto el índice Gleason - y el del tratamiento. En el grupo de bajo riesgo (Gleason menor o igual a 6) se produjeron 3 muertes por $\mathrm{CP}$, para 44 casos incluidos (7\%), es decir, se producen pocas muertes por CP y la supervivencia media es alta, hasta unos 16 años. En este análisis, es mayor la supervivencia observada en el grupo de la hormonoterapia - 17,38 años, frente a 15,33 años los no tratados -, aunque no existe diferencia estadísticamente significativa. La diferencia, considerando la edad media en el diagnóstico, no resulta muy relevante.

En el grupo de alto riesgo, (Gleason mayor o igual a 7) la observación es sensiblemente distinta. Se registraron 13 muertes en 29 personas $(45 \%)$, con una supervivencia inferior en aproximadamente un año en el grupo tratado, aunque sin significación estadística.

En conclusión, del presente estudio se desprende que, como numerosos autores afirman, dentro de la entidad clínica que actualmente etiquetamos como "CP estadio I" se incluyen tanto enfermedades con potencial alto para producir progresión y muerte en los pacientes, como hallazgos poco significativos, que dificilmente comprometerán la expectativa de vida de sus portadores. En conse- 
cuencia, el reto para la Urología y para la Anatomía Patológica está en separar los casos "que se comportarán de una forma agresiva de los que evolucionarán de una forma indolente" y diferenciar con precisión entre los varones que se beneficiará de un tratamiento de la enfermedad y el que solo obtendrá molestias y, presumiblemente, pero no considerada en este estudio, angustia y yatrogenia.

En este sentido, parece que el indicador Gleason discrimina adecuadamente entre los que deben ser tratados y los que no. Además debe de considerarse que, a la luz de la serie presentada, el tratamiento hormonoterápico no supone ningún beneficio claro frente a la abstención terapéutica vigilada.

En cuanto a la conveniencia de realizar determinaciones de PSA en varones sin sintomatología prostática, debe tenerse presente que, además de las reticencias presentadas por distintos autores y agencias, tan solo un $40 \%$ de las personas de nuestra serie presentaron marcadores de riesgo alto (Gleason mayor de 7). Considerando su edad y el hecho de tratarse de "incidentalomas", cabe suponer que una extensión de la prueba de PSA a varones más jóvenes o sin sintomatología miccional importante, encontraría un aún menor porcentaje de tumores $\mathrm{T} 1$ de alto riesgo, de manera que la rentabilidad diagnóstica de la prueba sería menor.

En caso de que el PSA detectado en una persona sin síntomas de CP fuera alto - $\mathrm{y}$ no hemos considerado en este estudio el punto de corte- la biopsia y la determinación del índice Gleason son el procedimiento que mejor determina la actitud a seguir. Ante Gleason bajos (igual o menor de 6), la abstención vigilada se ha mostrado una alternativa aceptable, mientras que ante índices altos (Gleason mayores de 7), la actitud debería ser ofrecer al paciente un tratamiento con más posibilidades de curación que la hormonoterapia, que parece no aumentar de ninguna manera las posibilidades de supervivencia

\section{REFERENCIAS}

1. Jemal A, Murray T, Samuels A, Ghafoor A, Ward E, Thun MJ. Cancer statistics, 2003. CA Cancer J Clin. 2003;53(1): 5-26.

2. Bray F, Sankila R, Ferlay J, Parkin DM. Estimates of cancer incidence and mortality in Europe in 1995. Eur J Cancer. 2002; 38(1):99-166.
3. Ruiz Liso JM, Sanz Anquela JM, Alfaro Torres J, Garcia Perez MA, Dodero de Solano E. Trends in urologic neoplasm mortality and incidence (prostate, bladder, and kidney). Soria, 19501988]. Arch Esp Urol. 1989;42(6):532-538.

4. Parkin DM, Läärä E, Muir CS. Estimates of the worldwide frequency of sixteen major cancers in 1980. Int J Cancer. 1988; 41(2): 184-197.

5. Herranz Amo F, Arias Funez F, Arrizabalaga Moreno M, Calahorra Fernandez FJ, Carballido Rodriguez J, Diz Rodriguez R et al. Prostate cancer in the Community of Madrid in the year 2000. IV. Treatment. Actas Urol Esp. 2003;27(6):418-427.

6. Zincke H, Oesterling JE, Blute ML, Bergstralh EJ, Myers RP, Barrett DM. Long-term (15 years) results after radical prostatectomy for clinically localized (stage T2c or lower) prostate cancer. J Urol. 1994;152(5 Pt 2):1850-1857.

7. Berenguer Sánchez, Páez Borda A, Luján Galán M. Despistaje en carcinoma prostático. Tratado de Oncología Urológica. Tomo II. Pág. 709-717. Sanidad y Ediciones, S.L. 2003

8. Breslow N, Chan CW, Dhom G, Drury RA, Franks LM, Gellei B, et al. Latent carcinoma of prostate at autopsy in seven areas. The International Agency for Research on Cancer, Lyons, France. Int J Cancer. 1977;20(5):680-688.

9. Scardino PT, Weaver R, Hudson MA. Early detection of prostate cancer. Hum Pathol. 1992;23(3):211-222.

10. Neal DE, Leung HY, Powell PH, Hamdy FC, Donovan JL. Unanswered questions in screening for prostate cancer. Eur J Cancer. 2000;36(10):1316-1321.

11. Warner J, Whitmore WF. Expectant management of clinically localized prostatic cancer. J Urol. 1994;152(5 Pt 2): 1761-1765.

12. Johansson JE, Adami HO, Andersson SO, Bergström R, Krusemo UB, Kraaz W. Natural history of localised prostatic cancer. A population-based study in 223 untreated patients. Lancet. 1989;1(8642):799-803.

13. Fleming C, Wasson JH, Albertsen PC, Barry MJ, Wennberg JE. A decision analysis of alternative treatment strategies for clinically localized prostate cancer. Prostate Patient Outcomes Research Team. JAMA. 1993;269(20):2650-2658.

14. Wilt TJ, Brawer MK. The Prostate Cancer Intervention Versus Observation Trial: a randomized trial comparing radical prostatectomy versus expectant management for the treatment of clinically localized prostate cancer. J Urol. 1994;152(5 Pt 2): 1910-1914.

15. Thompson IM, Pauler DK, Goodman PJ, Tangen CM, Lucia MS, Parnes HL et al. Prevalence of prostate cancer among men with a prostate-specific antigen level $<$ or $=4.0 \mathrm{ng}$ per milliliter. $\mathrm{N}$ Engl J Med. 2004;350(22):2239-2246. Erratum in: N Engl J Med. 2004;351(14):1470.

16. Stamey TA, Caldwell M, McNeal JE, Nolley R, Hemenez M, Downs J. The prostate specific antigen era in the United States is over for prostate cancer: what happened in the last 20 years?. J Urol. 2004;172(4 Pt 1):1297-1301.

17. Catalona WJ, Loeb S. The PSA era is not over for prostate cancer. Eur Urol. 2005;48(4):541-545.

18. Holmberg L, Bill-Axelson A, Helgesen F, Salo JO, Folmerz P, Haggman $\mathrm{M}$ et al. A randomized trial comparing radical prostatectomy with watchful waiting in early prostate cancer. $\mathrm{N}$ Engl J Med. 2002;347(11):781-789.

19. Fernandez Rosado E, Gomez Veiga F, Alvarez Castelo L, Ruibal Moldes M, Mosquera Reboredo JM, Gonzalez Martin M. Prevalence of incidental prostatic adenocarcinoma after suprapubic adenomectomy with or without previous prostatic biopsy. Arch Esp Urol. 2006;59(1):31-42.

Correspondencia autor: Dr. S. Napal Lecumberri

Servicio de Urología. Hospital de Navarra

C/ Irunlarrea 3 - 31008 Pamplona. Tel.: 34848422100

E-mail autor: s_napal @yahoo.es

Información artículo: Original - Cáncer de próstata

Trabajo recibido: enero 2007

Trabajo aceptado: febrero 2007 